PROCEEDINGS OF THE

AMERICAN MATHEMATICAL SOCIETY

Volume 139, Number 1, January 2011, Pages 331-344

S 0002-9939(2010)10554-3

Article electronically published on July 29, 2010

\title{
COEXISTENCE IN INTERVAL EFFECT ALGEBRAS
}

\author{
GEJZA JENČA
}

(Communicated by Marius Junge)

\begin{abstract}
Motivated by the notion of coexistence of effect-valued observables, we give a characterization of coexistent subsets of interval effect algebras.
\end{abstract}

\section{INTRODUCTION AND MOTIVATION}

Let $\mathbb{H}$ be a Hilbert space and let $\mathcal{S}(\mathbb{H})$ be the partially ordered abelian group of all bounded self-adjoint operators on $\mathbb{H}$ equipped with the usual order. We write $\mathcal{E}(\mathbb{H})$ for the closed interval $[0, I]_{\mathcal{S}(\mathbb{H})}=\{A \in \mathcal{S}(\mathbb{H}): 0 \leq A \leq I\}$, where $I$ is the identity operator on $\mathbb{H}$. The elements of $\mathcal{E}(\mathbb{H})$ are called Hilbert space effects.

From the algebraic point of view, $\mathcal{E}(\mathbb{H})$, equipped with addition, is the canonical example of an interval effect algebra [9, [1].

Let $(\Omega, \mathcal{A})$ be a measurable space. An observable is a mapping $\alpha: \mathcal{A} \rightarrow \mathcal{E}(\mathbb{H})$ such that $\alpha(\emptyset)=0, \alpha(\Omega)=1$ and for every pairwise disjoint system $\left\{X_{i}\right\}_{i \in \mathbb{N}}$ of measurable sets, $\alpha\left(\bigcup_{i \in \mathbb{N}} X_{i}\right)=\sum_{i \in \mathbb{N}} \alpha\left(X_{i}\right)$. That means that an observable is a normalized positive operator measure $\alpha: \mathcal{A}: \rightarrow \mathcal{E}(\mathbb{H})$.

In the unsharp observable approach to quantum mechanics, observables represent measurable quantities; see for example [3, 5].

Definition 1. Let $\left(\Omega_{1}, \mathcal{A}_{1}\right)$ and $\left(\Omega_{2}, \mathcal{A}_{2}\right)$ be measurable spaces. Observables $\alpha_{1}$ : $\mathcal{A}_{1} \rightarrow \mathcal{E}(\mathbb{H})$ and $\alpha_{2}: \mathcal{A}_{2} \rightarrow \mathcal{E}(\mathbb{H})$ are coexistent if there is a measurable space $(\Omega, \mathcal{A})$ and an observable $\alpha: \mathcal{A} \rightarrow \mathcal{E}(\mathbb{H})$ such that

$$
\operatorname{ran}\left(\alpha_{1}\right) \cup \operatorname{ran}\left(\alpha_{2}\right) \subseteq \operatorname{ran}(\alpha),
$$

where $\operatorname{ran}($.$) denotes the range of an observable.$

In mathematical physics, the notion of coexistence of observables is used to describe the possibility of measuring together two quantities. The concept of coexistence of observables is due to Ludwig [24] and it was further investigated in [13], [19, 23. See also 20], 22], 21], 26] for recent results on coexistence of observables and [12, 4, 29] for investigations concerning coexistence of qubit effects.

Obviously, the coexistence of any pair of observables depends only on their ranges; two observables are coexistent if and only if the union of their ranges can

Received by the editors September 26, 2009 and, in revised form, March 19, 2010.

2010 Mathematics Subject Classification. Primary 03G12; Secondary 06F20, 81P10.

Key words and phrases. Effect algebra, coexistent observables.

This research is supported by grant VEGA G-1/0080/10 of MŠ SR, Slovakia and by the Slovak Research and Development Agency under contract No. APVV-0071-06.

(C)2010 American Mathematical Society Reverts to public domain 28 years from publication 
be embedded into a range of an observable. This fact gives rise to the following problem.

Problem 1. Give a characterization of such sets $S$ of effects on a Hilbert space $\mathbb{H}$ such that there is a measurable space $(\Omega, \mathcal{A})$ and an observable $\alpha: \mathcal{A} \rightarrow \mathcal{E}(\mathbb{H})$ with $S \subseteq \operatorname{ran}(\alpha)$.

If $S$ consists only of orthogonal projections (which means, idempotent effects), then the answer is simple: $S$ is a subset of the range of an observable if and only if the elements of $S$ commute. On the other hand, if there are non-idempotent effects in $S$, the situation is much more complicated.

In the present paper, we examine a more general problem:

Problem 2. Let $E$ be an interval effect algebra. Characterize subsets $S$ of $E$ such that there is a Boolean algebra $B$ and a morphism of effect algebras $\alpha: B \rightarrow E$ with $S \subseteq \operatorname{ran}(\alpha)$.

This can be considered as an abstract algebraic version of Problem 1. In the present paper, we prove that, given a subset $S$ of an interval effect algebra $E$, there exist a Boolean algebra $B$ and a morphism $\alpha: B \rightarrow E$ with $S \subseteq \operatorname{ran}(\alpha)$ if and only if there is a mapping $\beta: \operatorname{Fin}(S) \rightarrow E$ satisfying certain properties. We call such mappings witness mappings. In [15, we introduced and studied a similar but more complicated notion, called compatibility support mappings, in the context of general effect algebras. In the present paper, the more restrictive setting of interval effect algebras allows us to introduce a simpler and much more intuitive notion of a witness map, and we can still apply our results to our most important example, namely $\mathcal{E}(\mathbb{H})$.

\section{Definitions And BASIC RElationships}

2.1. Effect algebras. An effect algebra is a partial algebra $(E ; \oplus, 0,1)$ with a binary partial operation $\oplus$ and two nullary operations 0,1 satisfying the following conditions:

(E1) If $a \oplus b$ is defined, then $b \oplus a$ is defined and $a \oplus b=b \oplus a$.

(E2) If $a \oplus b$ and $(a \oplus b) \oplus c$ are defined, then $b \oplus c$ and $a \oplus(b \oplus c)$ are defined and $(a \oplus b) \oplus c=a \oplus(b \oplus c)$.

(E3) For every $a \in E$ there is a unique $a^{\prime} \in E$ such that $a \oplus a^{\prime}$ exists and $a \oplus a^{\prime}=1$.

(E4) If $a \oplus 1$ is defined, then $a=0$.

Effect algebras were introduced by Foulis and Bennett in their paper 9 .

In their paper [18, Chovanec and Kôpka introduced an essentially equivalent structure called a $D$-poset. Their definition is an abstract algebraic version of the D-poset of fuzzy sets, introduced by Kôpka in the paper [17].

Another equivalent structure was introduced by Giuntini and Greuling in [11. We refer to [8] for more information on effect algebras and related topics.

The class of effect algebras is (essentially) a common superclass of several important classes of algebras: orthomodular lattices [16, 2], orthoalgebras [10, MValgebras [6, 25. In particular, every Boolean algebra is an effect algebra if we introduce a partial operation $\oplus$ such that $a \oplus b$ exists if and only if $a \wedge b=0$ and then we put $a \oplus b:=a \vee b$. 
2.2. Properties of effect algebras. In an effect algebra $E$, we write $a \leq b$ if and only if there is $c \in E$ such that $a \oplus c=b$. It is easy to check that for every effect algebra $E, \leq$ is a partial order on $E$. Moreover, it is possible to introduce a new partial operation $\ominus ; b \ominus a$ is defined if and only if $a \leq b$ and then $a \oplus(b \ominus a)=b$. It can be proved that, in an effect algebra, $a \oplus b$ is defined if and only if $a \leq b^{\prime}$ if and only if $b \leq a^{\prime}$. In an effect algebra, we write $a \perp b$ if and only if $a \oplus b$ exists.

A finite family $\left(a_{1}, \ldots, a_{n}\right)$ of elements of an effect algebra is called orthogonal if and only if the sum $a_{1} \oplus \cdots \oplus a_{n}$ exists. An orthogonal family $\left(a_{1}, \ldots, a_{n}\right)$ is a decomposition of unit if and only if $a_{1} \oplus \cdots \oplus a_{n}=1$.

2.3. Morphisms of effect algebras. Let $E, F$ be effect algebras. A mapping $\phi: E \rightarrow F$ is a morphism of effect algebras if and only if the following conditions are satisfied:

(EM1) $\phi(1)=1$.

(EM2) If $a, b \in E$ and $a \perp b$, then $\phi(a) \perp \phi(b)$ and $\phi(a \oplus b)=\phi(a) \oplus \phi(b)$.

We note that every morphism of effect algebras is isotone. Moreover, every morphism of effect algebras preserves the 0 element, as well as the unary operation $x \mapsto x^{\prime}$ and the partial binary operation $\ominus$.

A bijective morphism of effect algebras $\phi: E \rightarrow F$ such that $\phi^{-1}$ is a morphism of effect algebras is called an isomorphism of effect algebras.

Let $B$ be a Boolean algebra and let $E$ be an effect algebra. A morphism of effect algebras $\alpha: B \rightarrow E$ is called an observable. If $B$ is finite, then we say that $\alpha$ is a simple observable.

Definition 2. We say that a subset $S$ of an effect algebra is coexistent if there exists a Boolean algebra $B$ and an observable $\alpha: B \rightarrow E$ such that $S \subseteq \alpha(B)$.

If is easy to check that a subset $\{a, b\}$ of an effect algebra $E$ is coexistent if and only if there is $c \in E$ such that $c \leq a, b$ and $a \perp b \ominus c$. Following the terminology in [12, we say that $c$ is the witness element for $a, b$.

2.4. Partially ordered abelian groups. Let $G$ be an (additive) abelian group. We say that $G$ is a partially ordered abelian group if and only if $G$ is equipped with a partial order that is compatible with addition, which means, for all $a, b, t \in G$,

$$
a \geq b \Longrightarrow a+t \geq b+t .
$$

Example 1. Let $\mathbb{H}$ be a Hilbert space and let $\mathcal{S}(\mathbb{H})$ be the set of all bounded self-adjoint operators on $\mathbb{H}$. For $A, B \in \mathcal{S}(\mathbb{H})$, write $A \leq B$ if and only if, for all $x \in \mathbb{H},\langle A x, x\rangle \leq\langle B x, x\rangle$. Then $(\mathcal{S}(\mathbb{H}),+, 0)$ is a partially ordered abelian group.

For a partially ordered abelian group $G$, we write

$$
G^{+}=\{a \in G: a \geq 0\} .
$$

The elements of $G^{+}$are called positive. Obviously, $G^{+}$is a submonoid of $G$. Moreover, $G^{+}$is conical, which means, if $a, b \in G^{+}$and $a+b=0$, then $a=b=0$.

It is easy to see that there is a one-to-one correspondence between partial orders on $G$ and conical submonoids of $G$. 
2.5. Order units. Let $G$ be a partially ordered abelian group. We say that $u \in G^{+}$ is an order unit if and only if for every $a \in G$ there is $n \in \mathbb{N}$ such that $n . u \geq a$.

A pair $(G, u)$, where $G$ is a partially ordered abelian group and $u$ is an order unit of $G$ is called a unital group.

Let $\left(G_{1}, u_{1}\right),\left(G_{2}, u_{2}\right)$ be unital groups. A mapping $\phi: G_{1} \rightarrow G_{2}$ is a morphism of unital groups if and only if $\phi$ is a group homomorphism, $x \geq y$ implies $\phi(x) \geq \phi(y)$ and $\phi\left(u_{1}\right)=u_{2}$.

For a morphism of unital groups, we write $\phi:\left(G_{1}, u_{1}\right) \rightarrow\left(G_{2}, u_{2}\right)$.

Example 2. In $\mathcal{S}(\mathbb{H})$, the identity operator is an order unit.

2.6. Interval effect algebras. One can construct examples of effect algebras from an arbitrary partially ordered abelian group $(G, \leq)$ in the following way: Choose any positive $u \in G$; then, for $0 \leq a, b \leq u$, define $a \oplus b$ if and only if $a+b \leq u$ and put $a \oplus b=a+b$. With such a partial operation $\oplus$, the closed interval

$$
[0, u]_{G}=\{x \in G: 0 \leq x \leq u\}
$$

becomes an effect algebra $\left([0, u]_{G}, \oplus, 0, u\right)$. Effect algebras which arise from partially ordered abelian groups in this way are called interval effect algebras; see [1].

More generally, we say that $E$ is an interval effect algebra in the group $G$ if $E$ is of the form $[0, u]_{G^{+}}$or isomorphic to such an effect algebra.

Example 3. The prototype interval effect algebra is the standard effect algebra $\mathcal{E}(\mathbb{H})=[0, I]_{\mathcal{S}(\mathbb{H})}$.

2.7. MV-algebras and MV-effect algebras. An $M V$-algebra (cf. [6, 25]) is a $(2,1,0)$-type algebra $(M ; \boxplus, \neg, 0)$, such that $\boxplus$ satisfies the identities $(x \boxplus y) \boxplus z=$ $x \boxplus(y \boxplus z), x \boxplus y=y \boxplus x, x \boxplus 0=x, \neg \neg x=x, x \boxplus \neg 0=\neg 0$ and

$$
x \boxplus \neg(x \boxplus \neg y)=y \boxplus \neg(y \boxplus \neg x) .
$$

On every MV-algebra, a partial order $\leq$ is defined by the rule

$$
x \leq y \Longleftrightarrow y=x \boxplus \neg(x \boxplus \neg y) .
$$

In this partial order, every MV-algebra is a distributive lattice bounded by 0 and $\neg 0$.

An $M V$-effect algebra is a lattice ordered effect algebra $M$ in which, for all $a, b \in$ $M$,

$$
(a \vee b) \ominus a=b \ominus(a \wedge b)
$$

It is proved in [7] that there is a natural, one-to-one correspondence between MVeffect algebras and $\mathrm{MV}$-algebras given by the following rules. Let $(M, \oplus, 0,1)$ be an MV-effect algebra. Let $\boxplus$ be a total operation given by $x \boxplus y=x \oplus\left(x^{\prime} \wedge y\right)$. Then $\left(M, \boxplus,{ }^{\prime}, 0\right)$ is an MV-algebra. Similarly, let $(M, \boxplus, \neg, 0)$ be an MV-algebra. Restrict the operation $\boxplus$ to the pairs $(x, y)$ satisfying $x \leq \neg y$ and call the new partial operation $\oplus$. Then $(M, \oplus, 0, \neg 0)$ is an MV-effect algebra.

We note that every Boolean algebra is an MV-effect algebra.

According to [25, every MV-effect algebra is isomorphic to an interval $[0, u]_{G}$, where $G$ is a lattice-ordered group. Thus, every MV-effect algebra is an interval algebra. 
2.8. Group-valued measures and ambient groups. Let $E$ be an effect algebra and let $\left(G_{2}, u_{2}\right)$ be a unital group. A morphism of effect algebras from $E$ to the interval effect algebra $\left[0, u_{2}\right]_{G_{2}}$ is called a group-valued measure.

Proposition 1. 1] Let $E$ be an interval effect algebra. There exists a unital group $\left(G_{1}, u_{1}\right)$ such that $E=\left[0, u_{1}\right]_{G_{1}}, E$ generates $G_{1}$ and for every unital group $\left(G_{2}, u_{2}\right)$ and every group-valued measure $\phi: E \rightarrow\left[0, u_{2}\right]_{G_{2}}$, $\phi$ extends to a unique morphism of unital groups $\widehat{\phi}:\left(G_{1}, u_{1}\right) \rightarrow\left(G_{2}, u_{2}\right)$. The unital group $\left(G_{1}, u_{1}\right)$ is unique up to isomorphism.

The unital group $\left(G_{1}, u_{1}\right)$ from Proposition 1 is called the ambient group of $E$, denoted by $G(E)$.

Example 4. $\mathcal{S}(\mathbb{H})$ is the ambient group of $\mathcal{E}(\mathbb{H})$.

2.9. Möbius inversion theorem. We say that a partially ordered set $(P, \leq)$ is locally finite if and only if every closed interval

$$
[x, y]_{P}:=\{z \in P: x \leq z \leq y\}
$$

is a finite set.

Let $(P, \leq)$ be a locally finite partially ordered set. Define $I(P)$ to be the set of all pairs $(x, y) \in P \times P$ such that $(x \leq y)$.

There exists a unique function $\mu: I(P) \rightarrow \mathbb{Z}$ such that, for all $(x, y) \in I(P)$,

$$
\sum_{x \leq z \leq y} \mu(x, z)=\delta_{x, y}
$$

where $\delta_{x, y}$ is the Kronecker delta. We say that $\mu$ is the Möbius mapping of the poset $P$. We refer to the classical paper [27] and to the monograph 28] for more information on Möbius mappings and related topics.

Example 5. Let $S$ be a set and write $\operatorname{Fin}(S)$ for the set of all finite subsets of $S$. For the poset $(\operatorname{Fin}(S), \subseteq)$, we have $\mu(X, Z)=(-1)^{|X|+|Z|}$.

Theorem 1 (Möbius inversion formula). Let $G$ be an abelian group and let $f$ : $I(P) \rightarrow G$. Define

$$
f^{\leq}(x, y):=\sum_{x \leq z \leq y} f(z, y) .
$$

Then

$$
f(x, y)=\sum_{x \leq z \leq y} \mu(x, z) f \leq(z, y) .
$$

We say that $f(x, y)$ is the Möbius inversion of $f \leq$.

\section{Witness MAPPINGS}

Let $E$ be an interval effect algebra in a partially ordered abelian group $G$. Let $S \subseteq E$. Let us write $\operatorname{Fin}(S)$ for the set of all finite subsets of $S$. Obviously, $(\operatorname{Fin}(S), \subseteq)$ is a locally finite poset.

For every mapping $\beta: \operatorname{Fin}(S) \rightarrow G$, we define a mapping $D_{\beta}: I(\operatorname{Fin}(S)) \rightarrow G$. For $(X, A) \in I(\operatorname{Fin}(S))$, the value $D_{\beta}(X, A) \in G$ is given by the rule

$$
D_{\beta}(X, A):=\sum_{X \subseteq Z \subseteq A}(-1)^{|X|+|Z|} \beta(Z) .
$$


Note that there is an obvious connection to Möbius inversions: define $\hat{\beta}$ : $I(\operatorname{Fin}(S)) \rightarrow G$ by

$$
\hat{\beta}(X, A)=\beta(X) .
$$

Then $D_{\beta}$ is the Möbius inversion of $\hat{\beta}$ with respect to the poset $(\operatorname{Fin}(S), \subseteq)$. By the Möbius inversion formula we see that

$$
\beta(X)=\hat{\beta}(X, A)=\sum_{X \leq Z \leq A} D_{\beta}(Z, A),
$$

for any $A \supseteq X$. In particular, $A:=X$ yields $\beta(X)=D_{\beta}(X, X)$.

Lemma 1. Let $E$ be an interval effect algebra in a partially ordered abelian group $G$. Let $S$ be a subset of $E$ and let $\beta: \operatorname{Fin}(S) \rightarrow G$. For all $c \in S \backslash A$,

$$
D_{\beta}(X, A)=D_{\beta}(X, A \cup\{c\})+D_{\beta}(X \cup\{c\}, A \cup\{c\}) .
$$

Proof. The proof is purely formal. Let us rewrite

$$
D_{\beta}(X, A \cup\{c\})=\sum_{X \subseteq Z \subseteq A \cup\{c\}}(-1)^{|X|+|Z|} \beta(Z) .
$$

For any $Z$ in the sum, either $c \in Z$ or $c \notin Z$. If $c \in Z$, then $X \cup\{c\} \subseteq Z \subseteq A \cup\{c\}$. If $c \notin Z$, then $X \subseteq Z \subseteq A$. Consequently,

$$
\begin{aligned}
D_{\beta}(X, A \cup\{c\}) & =\sum_{X \subseteq Z \subseteq A}(-1)^{|X|+|Z|} \beta(Z)+\sum_{X \cup\{c\} \subseteq Z \subseteq A \cup\{c\}}(-1)^{|X|+|Z|} \beta(Z) \\
& =D_{\beta}(X, A)+\sum_{X \cup\{c\} \subseteq Z \subseteq A \cup\{c\}}(-1)^{|X|+|Z|} \beta(Z) .
\end{aligned}
$$

It remains to observe that

$$
\begin{aligned}
& \sum_{X \cup\{c\} \subseteq Z \subseteq A \cup\{c\}}(-1)^{|X|+|Z|} \beta(Z) \\
= & -\sum_{X \cup\{c\} \subseteq Z \subseteq A \cup\{c\}}(-1)^{|X \cup\{c\}|+|Z|} \beta(Z)=D_{\beta}(X \cup\{c\}, A \cup\{c\}) .
\end{aligned}
$$

Definition 3. Let $E$ be an interval effect algebra and let $S \subseteq E$.

We say that a mapping $\beta: \operatorname{Fin}(S) \rightarrow E$ is a witness mapping for $S$ if and only if the following conditions are satisfied:

(A1) $\beta(\emptyset)=1$,

(A2) for all $c \in S, \beta(\{c\})=c$,

(A3) for all $(X, A) \in I(\operatorname{Fin}(S)), D_{\beta}(X, A) \geq 0$.

Let us prove that our notion of a witness mapping can be considered as an extension of the notion of a witness element.

Proposition 2. Let $E$ be an interval effect algebra in a partially ordered abelian group $G$. Let $\{a, b\}$ be a subset of $E$ and let $\beta: \operatorname{Fin}(\{a, b\}) \rightarrow E$ be a mapping satisfying the conditions (A1) and (A2) of Definition 3 , Then $\beta$ is a witness map for $\{a, b\}$ if and only if $\beta(\{a, b\})$ is a witness for $a, b$.

Proof. Let the mapping $\beta: \operatorname{Fin}(\{a, b\}) \rightarrow E$ be given by

\begin{tabular}{c|c|c|c|c}
$X$ & $\emptyset$ & $\{a\}$ & $\{b\}$ & $\{a, b\}$ \\
\hline$\beta(X)$ & 1 & $a$ & $b$ & $c$
\end{tabular}.


Suppose that $c$ is a witness element. Let us prove the condition (A3). If $X=A$, there is nothing to prove. If $X=\emptyset$ and $A=\{x\}$, then $D_{\beta}(X, A)=1-x \geq 0$ since $x \leq 1$. If $X=\{x\}$ and $A=\{a, b\}$, then $D_{\beta}(X, A)=x-c \geq 0$, since $c \leq x$. If $X=\emptyset$ and $A=\{a, b\}$, then

$$
D_{\beta}(X, A)=D_{\beta}(\emptyset,\{a, b\})=1-a-b+c .
$$

As $c$ is a witness element for $a, b, a \ominus c \perp b$. This can be written as $a \ominus c \leq 1 \ominus b$. Therefore, $(1 \ominus b) \ominus(a \ominus c) \geq 0$, and we may compute in $G$ :

$$
(1 \ominus b) \ominus(a \ominus c)=1-b-a+c=1-a-b+c=D_{\beta}(\emptyset,\{a, b\}) .
$$

Thus, $D_{\beta}(\emptyset,\{a, b\}) \geq 0$.

Suppose that $\beta$ is a witness map. As $D_{\beta}(\{a\},\{a, b\})=a-c \geq 0$, we see that $a \geq c$. Similarly, $b \geq c$. As $D_{\beta}(\emptyset,\{a, b\})=1-a-b+c \geq 0$, the equality (4) implies that $a \ominus c \perp b$. Thus, $c$ is a witness element.

As an obvious consequence, we obtain the following.

Corollary 1. Let $\{a, b\}$ be a subset of an interval effect algebra E. Then $\{a, b\}$ is coexistent if and only if there is a witness mapping for $\{a, b\}$.

3.1. Properties of witness mappings. To shorten our formulations, let us introduce some running notation:

- $E$ is an interval effect algebra,

- $S$ is a subset of $E$,

- $\beta: \operatorname{Fin}(S) \rightarrow E$ is a witness mapping for $S$.

Let us prove that $D_{\beta}$ is, in fact, an $E$-valued mapping.

Proposition 3. For all $(X, A) \in I(\operatorname{Fin}(S)), D_{\beta}(X, A) \leq 1$.

Proof. By equation (3),

$$
\beta(X)=\hat{\beta}(X, A)=\sum_{X \leq Z \leq A} D_{\beta}(X, Z) .
$$

In particular, $D_{\beta}(X, A) \leq \beta(X) \leq 1$.

Since $D_{\beta}$ is an $E$-valued mapping, the + operation mentioned in Lemma 1 is a $\oplus$ partial operation of the effect algebra $E$ :

Proposition 4. For all $(X, A) \in I(\operatorname{Fin}(S))$ and $c \in S \backslash A, D_{\beta}(X, A \cup\{c\}) \perp$ $D_{\beta}(X \cup\{c\}, A \cup\{c\})$ and

$$
D_{\beta}(X, A)=D_{\beta}(X, A \cup\{c\}) \oplus D_{\beta}(X \cup\{c\}, A \cup\{c\}) .
$$

Proof. By Lemma 1

$$
D_{\beta}(X, A)=D_{\beta}(X, A \cup\{c\})+D_{\beta}(X \cup\{c\}, A \cup\{c\}) .
$$

By Definition 3 and Proposition 3, $D_{\beta}: I(\operatorname{Fin}(S)) \rightarrow E$.

Lemma 2. Let $X \in \operatorname{Fin}(S)$. If $1 \in S \backslash X$, then $D_{\beta}(X, X \cup\{1\})=0$.

Proof. The proof goes by induction with respect to $|X|$. If $X=\emptyset$, then

$$
D_{\beta}(X, X \cup\{1\})=D_{\beta}(\emptyset,\{1\})=\beta(\emptyset)-\beta(\{1\})=1-1=0 .
$$


Suppose that the lemma is true for some $X$ and let $c \notin X, c \neq 1$. We want to prove that $D_{\beta}(X \cup\{c\}, X \cup\{c\} \cup\{1\})=0$. Put $A=X \cup\{1\}$ in Proposition 4 to obtain

$$
D_{\beta}(X, X \cup\{1\})=D_{\beta}(X, X \cup\{c\} \cup\{1\}) \oplus D_{\beta}(X \cup\{c\}, X \cup\{c\} \cup\{1\}) .
$$

By the induction hypothesis, $D_{\beta}(X, X \cup\{1\})=0$, and since

$$
D_{\beta}(X, X \cup\{1\}) \geq D_{\beta}(X \cup\{c\}, X \cup\{c\} \cup\{1\}),
$$

we may conclude that $D_{\beta}(X \cup\{c\}, X \cup\{c\} \cup\{1\})=0$.

Later in Proposition 8 we will show that for every MV-effect algebra $M, \wedge$ : $\operatorname{Fin}(M) \rightarrow M$ is a witness mapping. The following proposition shows that several properties of $\bigwedge$ are preserved for all witness mappings.

\section{Proposition 5.}

(a) $\beta$ is an antitone mapping from $(\operatorname{Fin}(S), \subseteq)$ to $(E, \leq)$.

(b) For all $X \in \operatorname{Fin}(S), \beta(X)$ is a lower bound of $X$.

(c) Suppose that $0 \in S$. If $0 \in X \in \operatorname{Fin}(S)$, then $\beta(X)=0$.

(d) Suppose that $1 \in S$. For all $X \in \operatorname{Fin}(S), \beta(X)=\beta(X \cup\{1\})$.

Proof.

(a) Let $X \in \operatorname{Fin}(S)$. Let us prove that for any $c \in S \backslash X, \beta(X \cup\{c\}) \leq \beta(X)$. Put $X=A$ in Proposition 4 to obtain

$$
\begin{array}{r}
\beta(X)=D_{\beta}(X, X)=D_{\beta}(X, X \cup\{c\}) \oplus D_{\beta}(X \cup\{c\}, X \cup\{c\}) \\
\geq D_{\beta}(X \cup\{c\}, X \cup\{c\})=\beta(X \cup\{c\}) .
\end{array}
$$

The rest of the proof is a trivial induction.

(b) Let $c \in X$. By (a), $\{c\} \subseteq X$ implies that

$$
c=\beta(\{c\}) \geq \beta(X) .
$$

(c) Trivial, by (b).

(d) If $1 \in X$, there is nothing to prove.

Suppose that $1 \notin X$. Putting $A=X$ and $c=1$ in Proposition 4 yields

$$
D_{\beta}(X, X)=D_{\beta}(X, X \cup\{1\}) \oplus D_{\beta}(X \cup\{1\}, X \cup\{1\}) .
$$

By Lemma 2, $D_{\beta}(X, X \cup\{1\})=0$; hence

$$
\beta(X)=D_{\beta}(X, X)=D_{\beta}(X \cup\{1\}, X \cup\{1\})=\beta(X \cup\{1\}) .
$$

Proposition 6. Let $E_{1}, E_{2}$ be interval effect algebras. Let $\phi: E_{1} \rightarrow E_{2}$ be a morphism of effect algebras. If $S_{1} \subseteq E_{1}$ is such that there is a witness mapping $\beta_{1}$ of $S_{1}$, then $\phi\left(S_{1}\right)$ admits a witness mapping.

Proof. The mapping $\phi$ is a $G\left(E_{2}\right)$-valued measure on $E_{1}$. Therefore, there is a morphism of unigroups $\left.\widehat{\phi}:\left(G\left(E_{1}\right), 1\right) \rightarrow\left(G\left(E_{2}\right), 1\right)\right)$ extending $\phi$.

For every $a \in \widehat{\phi}\left(S_{1}\right)$, fix $p(a) \in S_{1}$ such that $\widehat{\phi}(p(a))=a$. Define $\beta_{2}: \operatorname{Fin}\left(\widehat{\phi}\left(S_{1}\right)\right)$ $\rightarrow E_{2}$ as follows:

$$
\beta_{2}\left(\left\{x_{1}, \ldots, x_{n}\right\}\right)=\widehat{\phi}\left(\beta_{1}\left(\left\{p\left(x_{1}\right), \ldots, p\left(x_{n}\right)\right\}\right)\right),
$$

or, in other words, for $X \in \operatorname{Fin}\left(S_{2}\right), \beta_{2}(X)=\widehat{\phi}\left(\beta_{1}(p(X))\right)$. Then $\beta_{2}$ is a witness mapping for $\widehat{\phi}\left(S_{1}\right)$. 
Indeed, the conditions (A1) and (A2) are trivially satisfied. For the proof of (A3) we may compute

$$
\begin{aligned}
& D_{\beta_{2}}(X, A)=\sum_{X \subseteq Z \subseteq A}(-1)^{|X|+|Z|} \beta_{2}(Z)=\sum_{X \subseteq Z \subseteq A}(-1)^{|X|+|Z|} \widehat{\phi}\left(\beta_{1}(p(Z))\right) \\
= & \widehat{\phi}\left(\sum_{X \subseteq Z \subseteq A}(-1)^{|X|+|Z|} \beta_{1}(p(Z))\right)=\widehat{\phi}\left(\sum_{p(X) \subseteq Y \subseteq p(A)}(-1)^{|p(X)|+|Y|} \beta_{1}(Y)\right) .
\end{aligned}
$$

Since $\beta_{1}$ is a witness mapping, $(-1)^{|p(X)|+|Y|} \beta_{1}(Y) \in E_{1}$. Therefore,

$$
\widehat{\phi}\left(\sum_{p(X) \subseteq Y \subseteq p(A)}(-1)^{|p(X)|+|Y|} \beta_{1}(Y)\right) \in E_{2} .
$$

Proposition 7. For every $S_{0} \subseteq S$, the restriction of $\beta$ to $\operatorname{Fin}\left(S_{0}\right)$ is a witness mapping for $S_{0}$.

Proof. Trivial.

\section{EXAMPLES OF WITNESS MAPPINGS}

To show that the notion of a witness mapping is natural, we present two examples of witness mappings, arising from the meet operation on an MV-effect algebra and the product operation on an commuting subset of $\mathcal{E}(\mathbb{H})$.

Proposition 8. Let $M$ be an $M V$-effect algebra. For the mapping $\bigwedge: \operatorname{Fin}(M) \rightarrow$ $M$,

$$
D_{\wedge}(X, A)=\bigwedge X \ominus((\bigwedge X) \wedge(\bigvee A \backslash X)) .
$$

Proof. The proof goes by induction with respect to $|A \backslash X|$.

If $|A \backslash X|=0$, then $A=X$ and

$$
D_{\wedge}(X, A)=D_{\wedge}(X, X)=\bigwedge X .
$$

For the right-hand side,

$$
\bigwedge X \ominus((\bigwedge X) \wedge(\bigvee A \backslash X))=\bigwedge X \ominus((\bigwedge X) \wedge 0)=\bigwedge X
$$

Let $n \in \mathbb{N}$ and suppose that the proposition is true for all pairs $(X, A)$ with $|A \backslash X| \leq n$. Let $X, A_{1} \in \operatorname{Fin}(M)$ be such that $X \subseteq A_{1}$ and $\left|A_{1} \backslash X\right|=n+1$. Pick $c \in A_{1} \backslash X$ and put $A:=A_{1} \backslash\{c\}$. Then $c \notin A$ and $A_{1}=A \cup\{c\}$.

By Lemma 1 .

$$
D_{\wedge}(X, A)=D_{\wedge}(X, A \cup\{c\})+D_{\wedge}(X \cup\{c\}, A \cup\{c\}) ;
$$

hence

$$
D_{\wedge}(X, A \cup\{c\})=D_{\wedge}(X, A)-D_{\wedge}(X \cup\{c\}, A \cup\{c\}) .
$$

To abbreviate, let us write $x:=\bigwedge X, a:=\bigvee A \backslash X$. Note that $\bigvee(A \cup\{c\}) \backslash(X \cup$ $\{c\})=a$ and that $\bigvee(A \cup\{c\}) \backslash X=a \vee c$. We need to prove that

$$
D_{\wedge}(X, A \cup\{c\})=x \ominus x \wedge(a \vee c) .
$$

By the induction hypothesis, we may write

$$
\begin{aligned}
D_{\wedge}(X, A) & =x \ominus x \wedge a, \\
D_{\wedge}(X \cup\{c\}, A \cup\{c\}) & =(x \wedge c) \ominus(x \wedge c \wedge a) .
\end{aligned}
$$


Therefore

$$
D_{\wedge}(X, A \cup\{c\})=(x \ominus x \wedge a)-((x \wedge c) \ominus(x \wedge c \wedge a)) .
$$

Thus, it remains to prove that

$$
x \ominus x \wedge(a \vee c)=(x \ominus x \wedge a)-((x \wedge c) \ominus(x \wedge c \wedge a)),
$$

which means that

$$
((x \wedge c) \ominus(x \wedge c \wedge a))+(x \ominus x \wedge(a \vee c))=x \ominus x \wedge a .
$$

Since $M$ is an MV-effect algebra, we may use the equality (1) and distributivity of $M$ as a lattice to compute

$$
\begin{aligned}
(x \wedge c) \ominus(x \wedge c \wedge a)=((x \wedge c) & \ominus((x \wedge c) \wedge(x \wedge a)))=((x \wedge c) \vee(x \wedge a)) \ominus(x \wedge a) \\
= & (x \wedge(c \vee a)) \ominus(x \wedge a)=x \wedge(a \vee c) \ominus(x \wedge a)
\end{aligned}
$$

hence

$$
\begin{array}{r}
((x \wedge c) \ominus(x \wedge c \wedge a))+x \ominus x \wedge(a \vee c) \\
=(x \wedge(a \vee c) \ominus(x \wedge a))+(x \ominus x \wedge(a \vee c))=x \ominus x \wedge a .
\end{array}
$$

Corollary 2. Let $M$ be an $M V$-effect algebra. The mapping $\bigwedge: \operatorname{Fin}(M) \rightarrow M$ is a witness mapping.

Proof. Clearly, the conditions (A1) and (A2) are satisfied.

Moreover, for any $(X, A) \in \operatorname{Fin}(M), \bigwedge X \geq((\bigwedge X) \wedge(\bigvee A \backslash X))$. By Proposition 8

$$
D_{\wedge}(X, A)=\bigwedge X \ominus((\bigwedge X) \wedge(\bigvee A \backslash X))
$$

Therefore, $D_{\wedge}(X, A) \geq 0$, and we see that (A3) is satisfied.

Corollary 3. Let $E$ be an interval effect algebra and let $M$ be an $M V$-effect algebra. Let $\phi: M \rightarrow E$ be a morphism of effect algebras. Every $S \subseteq \phi(M)$ admits a witness mapping.

Proof. By Corollary 2, $M$ admits a witness mapping. By Proposition 6, $\phi(M)$ admits a witness mapping. By Proposition $7, S$ admits a witness mapping.

Corollary 4. Every coexistent subset of an interval effect algebra admits a witness mapping.

Proof. Just let the MV-effect algebra $M$ of Corollary 3 be a Boolean algebra.

Another natural example of a witness mapping is given by the following proposition, which can be considered as a generalization of Theorem 2.2 of [12].

Proposition 9. Let $S$ be a pairwise commuting subset of $\mathcal{E}(\mathbb{H})$.

Let $\Pi: \operatorname{Fin}(S) \rightarrow \mathcal{E}(\mathbb{H})$ be given by

$$
\Pi\left(\left\{x_{1}, \ldots, x_{n}\right\}\right)=x_{1} \cdots x_{n} .
$$

Then $\Pi$ is a witness mapping. 
Proof. The proof goes by induction with respect to $|A \backslash X|$.

For $A=X, D_{\Pi}(X, A)=D_{\Pi}(X, X)=\Pi(X)$ and $0 \leq \Pi(X)$.

Let $n \in \mathbb{N}$. Suppose that, for all $A, X \in \operatorname{Fin}(E)$ such that $|A \backslash X|=n, 0 \leq$ $D_{\Pi}(X, A)$. Let $A_{1}, X \in \operatorname{Fin}(E)$ be such that $\left|A_{1} \backslash X\right|=n+1$. Pick $c \in A_{1} \backslash X$ and write $A=A_{1} \backslash\{c\}$. We see that $c \notin A$ and that $A_{1}=A \cup\{c\}$. We shall prove that $0 \leq D_{\Pi}(X, A \cup\{c\})$.

By Lemma 1 .

$$
D_{\Pi}(X, A \cup\{c\})=D_{\Pi}(X, A)-D_{\Pi}(X \cup\{c\}, A \cup\{c\}) .
$$

Let us compute

$$
\begin{array}{r}
D_{\Pi}(X \cup\{c\}, A \cup\{c\})=\sum_{X \cup\{c\} \subseteq Z \subseteq A \cup\{c\}}(-1)^{|X \cup\{c\}|+|Z|} \Pi(Z) \\
=\sum_{X \subseteq Y \subseteq A}(-1)^{|X \cup\{c\}|+|Y \cup\{c\}|} \Pi(Y \cup\{c\})=\sum_{X \subseteq Y \subseteq A}(-1)^{|X|+|Y|} \Pi(Y \cup\{c\}) \\
=c . \sum_{X \subseteq Y \subseteq A}(-1)^{|X|+|Y|} \Pi(Y)=c . D_{\Pi}(X, A) .
\end{array}
$$

Thus, by (5) we obtain

$$
D_{\Pi}(X, A \cup\{c\})=D_{\Pi}(X, A)-c \cdot D_{\Pi}(X, A)=(I-c) \cdot D_{\Pi}(X, A),
$$

where $I$ is the identity operator. By the induction hypothesis, $0 \leq D_{\Pi}(X, A)$; hence

$$
0 \leq(I-c) \cdot D_{\Pi}(X, A) .
$$

\section{MAin RESUlt}

The goal of this section is to show that every subset of an interval effect algebra that admits a witness mapping is coexistent. The main tools we shall use to achieve that goal are the following definition and theorem.

Definition 4 (Definition 1.10.36 of [8]). Let $E$ be an effect algebra.

(a) Let $(H, \leq)$ be a directed set and $\Omega_{i}$ be a finite set for each $i \in H$. Whenever $i, j \in H$ and $i \leq j$, let there be a mapping $g_{i, j}: \Omega_{j} \rightarrow \Omega_{i}$ and denote by $\mathcal{G}:=\left\{g_{i, j}: i \leq j\right\}$ the collection of all such mappings. The pair $\left(\left(\Omega_{i}\right)_{i \in H}, \mathcal{G}\right)$ is called a projective system of finite sets if the following conditions hold:

(i) $g_{i, i}$ is the identity map on $\Omega_{i}$,

(ii) $g_{i, j} \circ g_{j, k}=g_{i, k}$, whenever $i \leq j \leq k$.

(b) We say that $\left(\left(\Omega_{i}, \alpha_{i}\right), \mathcal{G}\right)$ is a projective system of simple observables if $\left(\left(\Omega_{i}\right)_{i \in H}, \mathcal{G}\right)$ is a projective system of finite sets and for each $i \in H$, $\alpha_{i}: 2^{\Omega_{i}} \rightarrow E$ is a simple observable such that the following compatibility condition holds:

(iii) For all $X \in 2^{\Omega_{i}}, \alpha_{i}(X)=\alpha_{j}\left(g_{i, j}^{-1}(X)\right)$ whenever $i \leq j$.

Theorem 2 (Theorem 1.10 .37 of [8]). Let $E$ be an effect algebra. A subset $S$ of $E$ is coexistent if and only if there is a projective system of simple observables $\left(\left(\Omega_{i}, \alpha_{i}\right), \mathcal{G}\right)$ such that for every $a \in S$ there is an $i \in H$ such that $a \in \operatorname{ran}\left(\alpha_{i}\right)$.

Let us start with showing that every witness mapping $\beta: \operatorname{Fin}(S) \rightarrow E$ gives rise to a family of simple observables $\alpha_{A}: 2^{2^{A}} \rightarrow E$, for every $A \in \operatorname{Fin}(S)$. 
Lemma 3. Let $C, A, X \in \operatorname{Fin}(S)$ be such that $X \subseteq A$ and $C \cap A=\emptyset$. Then $\left(D_{\beta}(X \cup Y, A \cup C)\right)_{Y \subseteq C}$ is an orthogonal family and

$$
\bigoplus_{Y \subseteq C} D_{\beta}(X \cup Y, A \cup C)=D_{\beta}(X, A) .
$$

Proof. The proof goes by induction with respect to $|C|$.

For $C=\emptyset$, the lemma is trivially true. Let $C$ be such that $|C|=n$ and let $c \in S, c \notin A \cup C$. Let us consider the family

$$
\left(D_{\beta}(X \cup Z, A \cup C \cup\{c\})\right)_{Z \subseteq C \cup\{c\}} .
$$

For each $Z \subseteq C \cup\{c\}$, either $c \in Z$ or $c \notin Z$, so either $Z=Y \cup\{c\}$ or $Z=Y$, for some $Y \subseteq C$. Therefore, we can write

$$
\begin{aligned}
& \left(D_{\beta}(X \cup Z, A \cup C \cup\{c\})\right)_{Z \subseteq C \cup\{c\}} \\
& \quad=\left(D_{\beta}(X \cup Y, A \cup C \cup\{c\}), D_{\beta}(X \cup Y \cup\{c\}, A \cup C \cup\{c\})\right)_{Y \subseteq C} .
\end{aligned}
$$

By Proposition 4 ,

$$
D_{\beta}(X \cup Y, A \cup C \cup\{c\}) \oplus D_{\beta}(X \cup Y \cup\{c\}, A \cup C \cup\{c\})=D_{\beta}(X \cup Y, A \cup C) .
$$

It only remains to apply the induction hypothesis to finish the proof.

Corollary 5. For every $A \in \operatorname{Fin}(S),\left(D_{\beta}(X, A)\right)_{X \subseteq A}$ is a decomposition of the unit.

Proof. By Lemma 3

$$
\bigoplus_{X \subseteq A} D_{\beta}(\emptyset \cup X, \emptyset \cup A)=D_{\beta}(\emptyset, \emptyset)=\beta(\emptyset)=1 .
$$

Corollary 6. For every $A \in \operatorname{Fin}(S)$, the mapping $\alpha_{A}: 2^{\left(2^{A}\right)} \rightarrow E$, given by

$$
\alpha_{A}(\mathbb{X})=\bigoplus_{X \in \mathbb{X}} D_{\beta}(X, A)
$$

is a simple observable.

Proof. The atoms of $2^{\left(2^{A}\right)}$ are of the form $\{X\}$, where $X \subseteq A$. By Corollary 5 , $\left(\alpha_{A}(\{X\}): X \subseteq A\right)$ is a decomposition of the unit; the remainder of the proof is trivial.

Theorem 3. Let $E$ be an interval effect algebra. $S \subseteq E$ admits a witness mapping if and only if $S$ is coexistent.

Proof. One implication is Corollary 4.

To prove the other implication, we shall apply Theorem 2 Let $\beta$ be a witness mapping:

- $H=\operatorname{Fin}(S)$, ordered by inclusion.

- For all $A \in H, \Omega_{A}:=2^{A}$.

- For $U, V \in H$ with $U \subseteq V, g_{U, V}: \Omega_{V} \rightarrow \Omega_{U}$ is given by the rule $g_{U, V}(X)=$ $X \cap U$ and $\mathcal{G}$ is the collection of all such $g_{U, V}$.

- For all $A \in H, \alpha_{A}: 2^{\Omega(A)} \rightarrow E$ is given by the rule

$$
\alpha_{A}(\mathbb{X})=\bigoplus_{X \in \mathbb{X}} D_{\beta}(X, A)
$$


Let us prove that $\left(\left(\Omega_{A}, \alpha_{a}\right), \mathcal{G}\right)$ is a projective system of simple observables. The conditions (i) and (ii) of Definition 4 are easy to check. By Corollary 7 , every $\alpha_{A}$ is an observable. Since

$$
\alpha_{\{a\}}(\{\{a\}\})=D_{\beta}(\{a\},\{a\})=\beta(\{a\})=a,
$$

every $a \in S$ is in the range of the observable $\alpha_{\{a\}}$. It remains to prove the condition (iii).

It is easy to see that

$$
g_{U, V}^{-1}(\mathbb{X})=\left\{X \cup C_{0}: X \in \mathbb{X} \text { and } C_{0} \subseteq(V \backslash U)\right\} .
$$

For all $\mathbb{X} \in 2^{\left(2^{U}\right)}$,

$$
\begin{aligned}
\alpha_{V}\left(g_{U, V}^{-1}(\mathbb{X})\right) & =\alpha_{V}\left(\left\{X \cup C_{0}: X \in \mathbb{X} \text { and } C_{0} \subseteq(V \backslash U)\right\}\right) \\
& =\bigoplus\left(D_{\beta}\left(X \cup C_{0}, V\right): X \in \mathbb{X} \text { and } C_{0} \subseteq(V \backslash U)\right) \\
& =\bigoplus_{X \in \mathbb{X}}\left(\bigoplus_{C_{0} \subseteq(V \backslash U)} D_{\beta}\left(X \cup C_{0}, B\right)\right) .
\end{aligned}
$$

Put $Y:=C_{0}, C:=V \backslash U$; by Lemma 3 ,

$$
\bigoplus_{C_{0} \subseteq(V \backslash U)} D_{\beta}\left(X \cup C_{0}, B\right)=D_{\beta}(X, U) .
$$

Therefore,

$$
\alpha_{V}\left(g_{U, V}^{-1}(\mathbb{X})\right)=\bigoplus_{X \in \mathbb{X}} D_{\beta}(X, U)=\alpha_{U}(\mathbb{X}),
$$

and the condition (iii) is satisfied.

All the conditions of Theorem 2 are satisfied. Therefore, $S$ is a coexistent subset of $E$.

Corollary 7. Every $M V$-effect algebra is a coexistent subset of itself.

Proof. By Corollary 2 and Theorem 3 ,

We note that Corollary 7 was already proved in [14, using a different method.

Corollary 8. Let $\mathbb{H}$ be a Hilbert space. Every pairwise commuting subset of $\mathcal{E}(\mathbb{H})$ is coexistent.

Proof. By Corollary 9 and Theorem 3

\section{REFERENCES}

[1] M.K. Bennett and D.J. Foulis. Interval and scale effect algebras. Advances in Applied Mathematics, 19:200-215, 1997. MR.1459498 (98m:06024)

[2] L. Beran. Orthomodular Lattices, Algebraic Approach. Kluwer, Dordrecht, 1985. MR784029 (86m:06015b)

[3] P. Busch, P. Lahti, and P. Mittelstaedt. The Quantum Theory of Measurement. SpringerVerlag, 2nd edition, 1996. MR1419313 (98b:81019)

[4] P. Busch and H-J. Schmidt. Coexistence of qubit effects. Quantum Information Processing, Quantum Information Processing 9:143-169, 2010.

[5] P. Bush, M. Grabowski, and P. Lahti. Operational Quantum Physics. Springer-Verlag, Berlin, 1995. MR $1356220(96 \mathrm{j}: 81022)$

[6] C.C. Chang. Algebraic analysis of many-valued logics. Trans. Amer. Math. Soc., 88:467-490, 1959. MR0094302 (20:821)

[7] F. Chovanec and F. Kôpka. Boolean D-posets. Tatra Mt. Math. Publ, 10:183-197, 1997. MR:1469294 (98c:03127) 
[8] A. Dvurečenskij and S. Pulmannová. New Trends in Quantum Structures. Kluwer, Dordrecht, and Ister Science, Bratislava, 2000. MR.1861369 (2002h:81021)

[9] D.J. Foulis and M.K. Bennett. Effect algebras and unsharp quantum logics. Found. Phys., 24:1325-1346, 1994. MR.1304942 (95k:06020)

[10] D.J. Foulis and C.H. Randall. Operational quantum statistics. I. Basic concepts. J. Math. Phys., 13:1667-1675, 1972. MR0416417 (54:4491)

[11] R. Giuntini and H. Greuling. Toward a formal language for unsharp properties. Found. Phys., 19:931-945, 1989. MR1013913 (90j:81017)

[12] S. Gudder. Coexistence of quantum effects. Reports on Mathematical Physics, 63(2):289-303, 2009. MR2519471

[13] K.-E. Hellwig. Coexistent effects in quantum mechanics. International Journal of Theoretical Physics, 2:147-155, 1969

[14] G. Jenča. Boolean algebras R-generated by MV-effect algebras. Fuzzy sets and systems, 145:279-285, 2004. MR2074002 (2005e:06018)

[15] G. Jenča. Compatibility support mappings in effect algebras. Math. Slovaca (to appear). arXiv:math.RA/0910.2825.

[16] G. Kalmbach. Orthomodular Lattices. Academic Press, New York, $1983 . \quad$ MR716496 (85f:06012)

[17] F. Kôpka. D-posets of fuzzy sets. Tatra Mt. Math. Publ., 1:83-87, $1992 . \quad$ MR1230466 (94e:04008)

[18] F. Kôpka and F. Chovanec. D-posets. Math. Slovaca, 44:21-34, 1994. MR1290269|(95i:03134)

[19] K. Kraus. States, Effects and Operations. Springer-Verlag, Berlin, $1983 . \quad$ MR725167 $(86 \mathrm{j}: 81008)$

[20] P. Lahti and S. Pulmannová. Coexistent observables and effects in quantum mechanics. Reports on Mathematical Physics, 39:339-351, 1997. MR1477898(98i:81013)

[21] P. Lahti and S. Pulmannová. Coexistence vs. functional coexistence of quantum observables. Reports on Mathematical Physics, 47:199-212, 2001. MR1836331(2002d:81018)

[22] P. Lahti, S. Pulmannová, and K. Ylinen. Coexistent observables and effects in convexity approach. Reports on Mathematical Physics, 39:6364-6371, 1998. MR1656976 (99j:81020)

[23] G. Ludwig. Foundations of Quantum Mechanics. Springer-Verlag, Berlin, 1983. MR690770 (85g:81014)

[24] Günther Ludwig. Versuch einer axiomatischen Grundlegung der Quantenmechanik und allgemeinerer physikalischer Theorien. Zeitschrift für Physik A Hadrons and Nuclei, 181(3):233260, 1964. MR0181244 (31:5473)

[25] D. Mundici. Interpretation of $\mathrm{AF} C^{*}$-algebras in Łukasziewicz sentential calculus. J. Functional Analysis, 65:15-53, 1986. MR819173 (87k:46146)

[26] S. Pulmannová. Compatibility and decompositions of effects. Journal of Mathematical Physics, 43:2817-2830, 2002. MR.1893702 (2003b:81012)

[27] Gian-Carlo Rota. On the foundations of combinatorial theory. I. Theory of Möbius Functions. Probability Theory and Related Fields, 2:340-368, 1964. MR0174487 (30:4688)

[28] Richard P. Stanley. Enumerative combinatorics, volume 1. Wadsworth and Brooks/Cole, Monterey, CA, 1986. MR0847717 (87j:05003)

[29] P. Stano, D. Reitzner, and T. Heinosaari. Coexistence of qubit effects. Phys. Rev. A, 78:012315, 2008. MR2491108 (2010d:81079)

Department of Mathematics and Descriptive Geometry, Faculty of Civil Engineering, Slovak University of Technology, Radlinského 11, Bratislava 813 68, Slovak REPUBLIC

E-mail address: gejza.jenca@stuba.sk 